'Laboratorio de Hemodinamia, Hospital Guillermo Grant Benavente, Concepción, Chile. ${ }^{2}$ Facultad de Medicina, Universidad de Concepción, Concepción, Chile.

Trabajo no recibió financiamiento.

Los autores declaran no tener conflictos de interés.

Recibido el 28 de septiembre de 2020, aceptado el 2 de febrero de 2021.

Correspondencia a:

Dr. Luis Pérez Pino Laboratorio de Hemodinamia Hospital Guillermo Grant Benavente.

San Martin 1436, Concepción, Chile. I.perezp@yahoo.es

\section{Sobrevida alejada de los pacientes con estenosis aórtica severa tratados con implante valvular percutáneo}

\author{
LUIS PÉREZ ${ }^{1,2}$, REINALDO VENEGAS ${ }^{1}$, GUILLERMO IBIETA ${ }^{1}$, \\ EDUARDO LECANNELIER ${ }^{12}$, ALECK STOCKINS ${ }^{1}$, \\ EUGENIO SANHUEZA ${ }^{1}$, FABRIZIO FASCE ${ }^{1,2}$, ALICEX GONZÁLEZ ${ }^{1}$, \\ JATÚN SÁEZ ${ }^{1,2}$, CONSTANZA SANDOVAL ${ }^{1}$, \\ VIRGINIA SEGALL ${ }^{1}$, ISABEL ROBLES ${ }^{1}$
}

\section{Long-term survival of patients with severe aortic stenosis undergoing transcatheter aortic valve implantation}

Background: Transcatheter aortic valve implantation (TAVI) is an effective and safe option for low, medium and high-risk patients with severe aortic stenosis (SAS). Aim: To analyze the clinical results and long-term survival of TAVI in our center. Material and Methods: Prospective analysis of 53 patients aged $73 \pm$ 10 years with a Society of Thoracic Surgeons (STS) score of $7.3 \pm 3.9 \%$. Results: In $96 \%$ a transfemoral access was used and, in most patients, ProGlides ${ }^{\mathrm{TM}}$ as vascular closure device was used. General anesthesia and conscious sedation were used in 79 and $21 \%$ of cases, respectively. Fifty-three valves were implanted, 42 self-expandable (SEV) and 11 balloon-expandable (Edwards Sapiens). The implant was successful in 49 patients (92,4\%). The transaortic gradient after TAVI was almost zero $\mathrm{mmHg}$ in all patients and one had a severe aortic regurgitation. Permanent pacemakers were needed in $17 \%$ of patients. Two patients had a pericardial effusion, and one had a major vascular complication. No strokes were recorded, and 30-day mortality was 3.7\%. At long-term follow up (23.4 \pm 21.6 months) the global survival was $85 \%$ and the rate of cardiovascular mortality was 5.9\%. Conclusions: In this series of intermediate to high-risk patients, TAVI was associated with an excellent early and long-term survival.

(Rev Med Chile 2021; 149: 514-519)

Key words: Aortic Valve Stenosis; Heart Valve Disease; Transcatheter Aortic Valve Implantation.
E limplante percutáneo de una válvula aórtica (TAVI) en pacientes con estenosis aórtica (EA) severa sintomática ha demostrado ser superior al tratamiento médico en pacientes considerados inoperables y/o de muy alto riesgo quirúrgico $^{1-4}$. Los resultados en los pacientes de menor riesgo han sido al menos comparables con la cirugía de reemplazo valvular aórtico (RVA). Los estudios PARTNER II ${ }^{5}$ y Core Valve SURTAVI Trial $^{6}$ que utilizaron válvulas balónexpandibles y autoexpandibles respectivamente, enrolaron pacientes de riesgo intermedio (STS entre 4 y 8 ) y demostraron que TAVI tiene una tasa de mortalidad similar a la cirugía con una menor incidencia de complicaciones neurológicas, de fibrilación auricular y de transfusiones, pero se asocia a una mayor tasa de complicaciones vasculares, necesidad de marcapasos definitivo y de regurgitación aórtica moderada o severa. Los resultados de las experiencias nacionales no son del todo conocidos 
y en vista de la necesidad creciente en conocer y desarrollar este procedimiento creemos necesario compartir nuestra experiencia inicial.

El objetivo de este estudio es conocer los resultados del implante percutáneo de una válvula aórtica en nuestro centro, con énfasis en la sobrevida a corto y largo plazo.

\section{Método}

Es un estudio prospectivo, observacional que incluyó un total de 53 pacientes con EA severa sintomática, sometidos a TAVI en el Hospital Regional de Concepción, desde agosto de 2012 a agosto de 2019.

El diagnóstico clínico fue confirmado con ecocardiograma transtorácico en todos los pacientes y con estudio hemodinámico invasivo en casos seleccionados. Los criterios de severidad fueron universalmente publicados ${ }^{7}$ y la decisión de un implante percutáneo fue discutida por un equipo multidisciplinario que incluyó cardiólogos clínicos, intervencionistas, cirujanos y anestesistas. Todos los pacientes fueron estudiados con angiotomografía de tórax, abdomen y pelvis y con ello se determinó el tamaño de la válvula, el número de velos, grado de calcificación del anillo, velos y tracto de salida del ventrículo izquierdo, el acceso vascular y las proyecciones de implante. Uso de software dedicado Prosize de Horos.

El procedimiento fue bajo anestesia general y/o sedación según las condiciones del paciente. Utilizamos válvulas autoexpandibles (Evolut ${ }^{\mathrm{TM}} \mathrm{R}$, Evolut ${ }^{\mathrm{TM}} \mathrm{PRO}$ y Portico ${ }^{\mathrm{TM}}$ ) y balón expandibles (Edwards Sapiens 3). La predilatación con balón fue evaluada caso a caso pero fue mandatoria en pacientes con gradientes $>100 \mathrm{mmHg}$ y en vávulas bicúspides. Se utilizó ecocardiograma transesofágico solo cuando hubo anestesia general.

En todos los pacientes el implante fue bajo estimulación de marcapasos a alta frecuencia $(>140$ $\mathrm{lpm}$ ) con sonda transvenosa en el ventriculo derecho o a través de la guía metálica de alto soporte usada en el implante. La elección de una u otra fue basada en la presencia de los factores de riesgo para bloqueo auriculoventricular completo post TAVI. Durante el implante utilizamos antigcoagulación completa con heparina no fraccionada y ACT de 300. Todos recibieron terapia antiplaquetaria dual por 3 meses.
El éxito del procedimiento fue definido como el combinado de ausencia de muerte durante el implante, una sola válvula correctamente situada con gradiente transaórtico $\leq 20 \mathrm{mmHg}$ y en ausencia de insuficiencia aórtica moderada o severa.

Los pacientes fueron monitorizados en unidad coronaria por 24-48 h. Todos los pacientes fueron seguidos desde el punto de vista clínico y ecocardiográfico.

Post implante inmediato evaluamos el gradiente transaórtico, la presencia de regurgitación aórtica, trastornos del ritmo, derrame pericárdico, complicaciones neurológicas, vasculares y la mortalidad. A largo plazo estudiamos la muerte por cualquier causa, presencia de fuga paravalvular y el implante de marcapaso permanente (MP).

La definición de éxito del procedimiento, muerte cardiovascular, muerte de todas las causas y complicaciones fue de acuerdo al Consenso sobre investigación en válvulas VARC $2^{9}$.

El objetivo primario fue la tasa de sobrevida a los 30 días y en el seguimiento alejado. El promedio de seguimiento fue 23,43 $\pm 21,6$ meses.

Los datos son expresados como promedio \pm desviación estandar en caso de variables continuas y como número y porcentage en variables categóticas.

Para el análisis de sobrevida se utilizó KaplanMeier. Todos los análisis se hicieron con el programa SPSS, versión 22.

Todos los pacientes firmaron consentimiento informado.

\section{Resultados}

El promedio de edad fue de 73,2 \pm 9,9 años y 35 fueron hombres (66\%). Las caracteristicas clínicas se describen en la Tabla 1. El STS score fue $7,3 \pm 3,9 \%$, sin embargo, $20 \%$ del grupo tenía STS $>8 \% ; 48$ pacientes tenían válvula tricúspide y 5 eran bicúspides. En relación al acceso vascular para el implante, en 51pacientes fue transfemoral, 1 caso transapical y 1 aórtico directo. Todos los accesos femorales, excepto uno, fueron percutáneos cerrados con dispositivos ProGlides ${ }^{\mathrm{TM}}$. Utilizamos anestesia general 42 (79\%) pacientes y sedación consciente con técnica minimalista en once. 53 válvulas fueron implantadas, 42 autoexpandibles y 11 balón expandible (Tabla 1).

El procedimiento fue exitoso en $92,4 \%$. Tres $(5,6 \%)$ pacientes tuvieron una complicación 
grave; 2 casos de rotura de la raíz aórtica una de ellas reparada de manera quirúrgica y 1 caso de rotura de arteria iliaca común que requirió cirugía vascular (Tabla 2). Solo un paciente con

\section{Tabla 1, Características generales de los} pacientes y del procedimiento TAVI

\begin{tabular}{|lc|}
\hline & $\mathbf{n}=\mathbf{5 3}$ \\
\hline Característica, \% & \\
Edad, años & $73,2 \pm 9,9$ \\
Hombres & 66 \\
STS score & $7,3 \pm 3,9$ \\
Fracción eyección & $56 \pm 15$ \\
Diabetes mellitus & 33,9 \\
AVE previo & 1,8 \\
Enfermedad coronaria & 39,6 \\
NYHA III-IV & 90 \\
Fibrilación auricular & 18,8 \\
Marcapasos previo & 9,4 \\
Válvula tricúspide & 90,5 \\
Procedimiento, \% & \\
Anestesia general & 79 \\
Sedación consciente & 21 \\
Acceso transfemoral & 96,2 \\
Predilatación & 22,6 \\
Cierre vascular Proglide ${ }^{\text {TM }}$ & 98 \\
Tipo de válvula, \% & \\
Auto-expandible & 21 \\
Core Valve & 79 \\
Evolut R & 19 \\
Evolut PRO & 52,3 \\
Balón-expandico & 26,1 \\
& 2,3 \\
\hline
\end{tabular}

insuficiencia aórtica moderada/severa y no hubo complicaciones neurológicas.

En relación a los trastornos del ritmo post TAVI, 8 de $48(16,6 \%)$ pacientes que no tenían MP previo requirieron implante de MP post TAVI, de los cuales dos tercios fueron implantados entre los primeros 15 pacientes de nuestro programa. La incidencia de MP entre los pacientes 16 y 53 es solo de 7,5\%.

En la Figura 1 se observan los cambios hemodinámicos postimplante. Existe una caída significativa en el gradiente transvalvular desde un promedio de 50,6 \pm 9,7 a 2,9 $\pm 4,7 \mathrm{mmHg}$ en el estudio inmediato $(\mathrm{p}<0,001)$. El seguimiento con ecocardiograma trabstorácico a los 6 y 24 meses demuestra un sostenido bajo gradiente transaórtico $(10 \pm 4 \mathrm{mmHg})$.

\section{Tabla 2. TAVI: Procedimiento y complicaciones inmediatas}

\begin{tabular}{lc}
\hline $\begin{array}{l}\text { Resultados inmediatos } \\
\text { Éxito del procedimiento, } \mathrm{n}(\%)\end{array}$ & $49(92,4)$ \\
Válvulas implantadas, (n) & 53 \\
Gradiente post TAVI, mmHg & $2,9 \pm 4,7$ \\
Complicaciones, $\mathbf{n}(\%)$ & \\
Rotura del anillo & $1(1,8)$ \\
Hematoma del anillo & $1(1,8)$ \\
Muerte & $1(1,8)$ \\
Derrame pericárdico & $2(3,7)$ \\
Complicación vascular mayor & $1(1,8)$ \\
Marcapasos permanente & $8(16,6)$ \\
Insuficiencia aórtica leve & $4(7,5)$ \\
Insuficiencia aórtica severa & $1(1,8)$
\end{tabular}

$\mathrm{mmHg}$

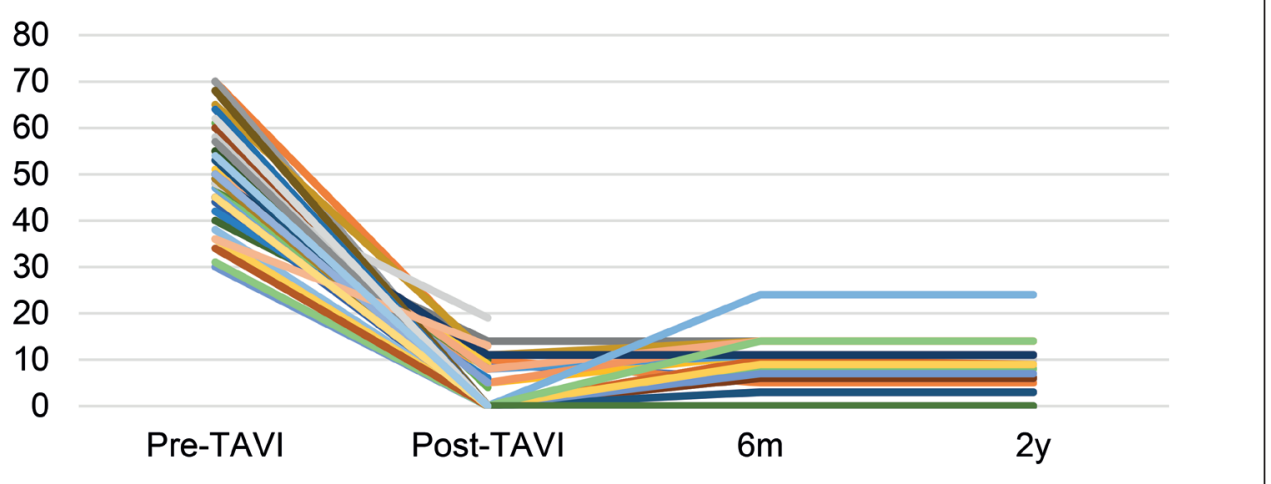

Figura 1. Variaciones en el gradiente transvalvular pre y post TAVI, expresados en mmHg. Resultados inmediatos y a largo plazo. 


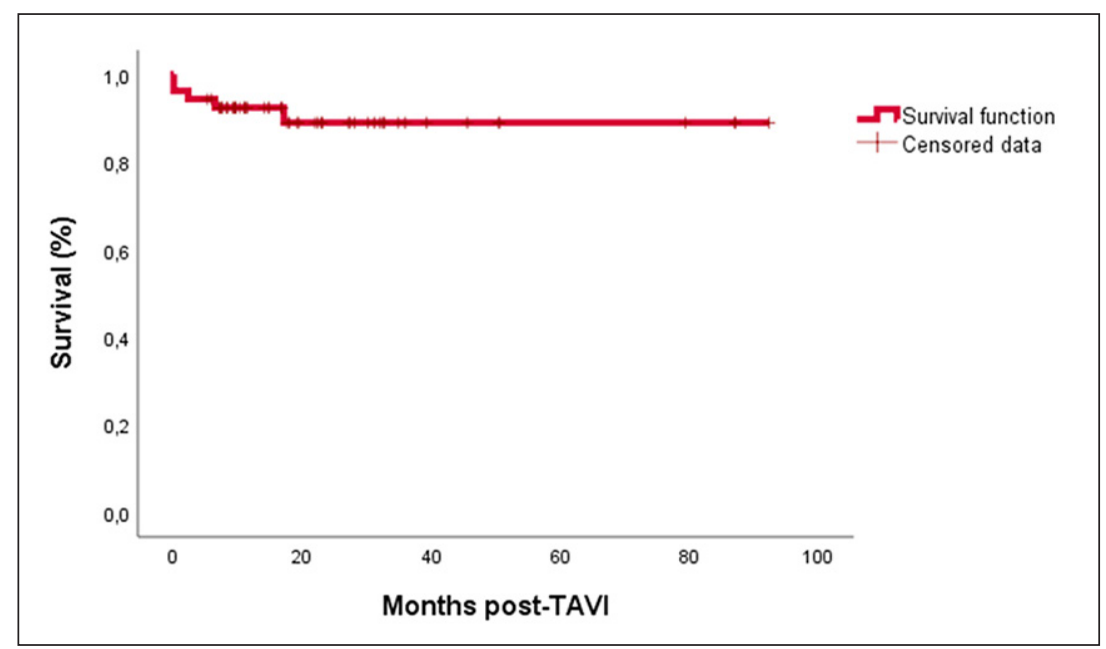

Figura 2. Curva de KaplanMeier para supervivencia libre de muerte.
La sobrevida a 30 días fue de $96,3 \%$ y a 2 años fue de un $85 \%$. Durante el seguimiento alejado, fallecieron $6(11,7 \%)$ pacientes, 3 de ellos debido a una causa cardiovascular, correspondiente al $5,9 \%$ (Figura 2). Todos los pacientes estaban en capacidad funcional I o II $(\mathrm{p}<0,001)$.

\section{Discusión}

Los hallazgos más importantes de nuestro estudio son: el implante percutáneo de una válvula aórtica tiene una alta tasa de éxito y se asocia a una excelente sobrevida al corto y largo plazo, la tasa de complicaciones en nuestra serie es muy baja y comparable a otros grupos internacionales y por último, aunque la incidencia de MP post TAVI fue similar a lo publicado, en nuestra serie hubo una clara relación con la curva de aprendizaje.

La estenosis aórtica severa es una de las valvulopatías más frecuente en la práctica clínica actual y en paralelo al envejecimiento de la población cada vez son más los pacientes adultos mayores que van a requerir de una intervención sobre la vávula aórtica. La edad, junto con la prevalencia de otras patologías concomitantes y la fragilidad de algunos pacientes hacen que el riesgo de mortalidad y morbilidad de los mismos no sea homogéneo. No existe dudas que la TAVI es superior a la cirugía en los pacientes de muy alto riesgo, consitituyendo una indicación clase I en la recomendaciones de Americana y Europea de Cardiología ${ }^{8,10}$. Además, múltiples estudios randomizados y observacionales han demostrado que la TAVI es no inferior al RVA en términos de mortalidad en pacientes de riesgo intermedio ${ }^{5,6,11-14}$.

La serie que publicamos representa un grupo de pacientes con EAS de riesgo intermedio tratados de manera percutánea con dispositivos predominantemente autoexpandibles. El STS fue $7,3 \pm 3,9 \%$ y el resultado más importante es la mortalidad de $3,7 \%$ a 30 días, mortalidad global de $15 \%$ a 2 años de seguimiento, con tan solo 5,9\% de mortalidad cardiovascular. Aun cuando es una serie pequeña y representa nuestra experiencia inicial, estos resultados son comparables a grandes series internacionales. En el estudio PARTNER II ${ }^{5}$, con 2032 pacientes en total, STS de 5,8 y el válvula balónexpandible SAPIEN X, la mortalidad en el grupo TAVI fue $3,9 \%$ y $16,7 \%$ a 30 días y 2 años, respectivamente. Los resultados de CoreValve (prótesis autoexpandible), mayoritaria en nuestra experiencia, en pacientes con riesgo intermedio fueron explorados en el estudio SURTAVI ${ }^{6} .1 .660$ pacientes con EAS, STS 4,4\% fueron randomizados a TAVI vs cirugía. La mortalidad a 30 días del grupo TAVI fue 2,2\% vs 1,7\% en el grupo quirúrgico, con una mortalidad de $11 \%$ a 2 años. En el metaanálisis de Sardar y cols ${ }^{15}$, que incluyó dos estudios randomizados y 5 observacionales, con un total de 4.600 pacientes en riesgo intermedio, la mortalidad global y cardiovascular es similar entre TAVI y RVA. Al igual que nosotros, la mortalidad fue de $4 \%$ a 30 días y $14,7 \%$ a 1,5 años. 
Las grandes series han reportado una tasa de accidentes cerebrovasculares entre $4,5-6 \%{ }^{5,6}$, sin embargo, en nuestra serie ningún paciente ha experimentado alguno.

Debido a la patogenia de la EA y a los mecanismos de implante, muy distintos al reemplazo quirúrgico, las principales preocupaciones respecto a TAVI en pacientes de riesgo intermedio o bajo, son la presencia de una insuficiencia aórtica (IA) severa y la necesidad de marcapasos definitivo. Respecto a la IA, en nuestra serie solo un paciente con gran calcificación del anillo quedó con IA severa que fue cerrada parcialmente con un dispositivo Amplatzer. Dicho paciente lleva dos años de seguimiento y sin hospitalizaciones. La complicación más frecuente ha sido el implante de un MP, con una incidencia del 16\%, similar a los descrito en la literatura ${ }^{4,6}$. Sin embargo, merece la pena comentar algunos puntos. La tasa MP en este estudio puede ser explicada en parte por el uso de prótesis autoexpandibles (CoreValve y Evolut) que conocidamente tienen mayor incidencia de trastornos de conducción comparados con las válvulas balónexpandibles, pero además está el factor relacionado a la falta de experiencia. En nuestra serie, 5 de los 8 marcapasos que hemos indicado post TAVI ocurrieron en los primeros 15 pacientes y desde entonces la tasa ha sido solo de 7,5\%. Esto puede ser atribuido a múltiples causas. Primero, hemos mejorado la profundidad de los implantes dejando las prótesis a no más de $3 \mathrm{~mm}$ respecto al anillo; segundo, hemos aprendido que no todos los trastornos de la conducción observadas durante el implante son indicación de MP y por último, hubo cambios en la técnica de implantes que han demostrado asociarse a menor tasa de trastornos de conducción y de MP. Del implante clásico con alineamiento fluoroscópico de los senos no coronariano, derecho e izquierdo nos hemos ido moviendo al implante de la sobreposición de senos coronarios (Cusp Overlap Techique), en donde se aisla el seno no coronariano y se sobreponen el derecho e izquierdo. Ello permite un implante más superficial y de esta forma menos contacto del la vávula protésica con el aparato de conducción. Diversos grupos han demostrado una notable menor incidencia de tratornos de conducción y necesidad de marcapasos con esta técnica. En un registro de latinoamericano presentado recientemente, en el cual nuestro centro participa, la incidencia de marcapasos fue $5,7 \%{ }^{16}$. De igual forma,
Gada y cols, en 169 pacientes que recibieron una válvula autoexpandible No34, grupo considerado de mayor riesgo de MP, usando técnica de overlap tuvieron una tasa de MP de $5,2 \%$ a 30 días ${ }^{17}$.

Finalmente, en paralelo al seguimiento clínico, los controles con ecocardiograma demuestran un excelente perfil hemodinámico de las prótesis a 2 años. Como se observa en la Figura 1, el gradiente promedio al final del seguimiento es de $10 \pm 4$ $\mathrm{mmHg}$. En solo un paciente, con válvula SAPIEN, hubo un severo aumento del gradiente a pocos meses que fue atribuido a trombosis de los velos y que revirtió con anticoagulación oral. Hoy en día, el gradiente residual así como el área efectiva de la válvula son importantes marcadores que hablarán sobre la durabilidad de las mismas ${ }^{18,19}$.

\section{Limitaciones}

La principal limitación de este estudio es su diseño observacional y descriptivo junto al tamaño de la muestra, lo que involucra sesgos de selección y por ello los resultados y conclusiones son débiles comparados con los estudios randomizados. Sin embargo, tiene tres grandes fortalezas. Lo primero es que representa a los pacientes de la vida real en donde tanto el médico tratante, el paciente y su familia participan de la selección del tratamiento; en segundo lugar posee un estricto seguimiento alejado y por último es una contribución para conocer la experiencia chilena en TAVI.

En conclusión, en esta cohorte de pacientes con estenosis aórtica severa de riesgo intermedio y alto para la cirugía, el implante de una prótesis aórtica percutánea es un tratamiento exitoso y seguro, con resultados comparables a grandes series internacionales.

\section{Referencias}

1. León MB, Smith CR, Mack M, Miller DC, Moses JW, Svensson LG, et al. Transcatheter aortic-valve implantation for aortic stenosis in patients who cannot undergo surgery. N Engl J Med. 2010; 363: 1597-607.

2. Popma JJ, Adams DH, Reardon MJ, Yakubov SJ, Kleiman NS, Heimansohn D, et al. Transcatheter aortic valve replacement using a self-expanding bioprosthesis in patients with severe aortic stenosis at extreme risk for surgery. J Am Coll Cardiol. 2014; 63: 1972-81. 
3. Smith CR, Leon MB, Mack MJ, Miller DC, Moses JW, Svensson LG, et al. Transcatheter versus surgical aortic-valve replacement in high-risk patients. $\mathrm{N}$ Engl J Med. 2011; 364: 2187-98.

4. Adams DH, Popma JJ, Reardon MJ, Yakubov SJ, Coselli JS, Deeb GM, et al. Transcatheter aortic-valve replacement with a self-expanding prosthesis. N Engl J Med. 2014; 370: 1790-8.

5. León MB, Smith CR, Mack MJ, Makkar RR, Svensson LG, Kodali SK, et al. PARTNER 2 Investigators. Transcatheter or Surgical Aortic-Valve Replacement in Intermediate-Risk Patients. N Engl J Med. 2016; 374 (17): 1609-20.

6. Reardon MJ, Van Mieghem NM, Popma JJ, Kleiman NS, Søndergaard L, Mumtaz M, et al. Surgical or transcatheter aortic-valve replacement in intermediate-risk patients. N Engl J Med. 2017; 376: 1321-31.

7. Nishimura RA, Otto CM, Bonow RO, Carabello BA, Erwin JP, Guyton RA, et al. 2014 AHA/ACC guideline for the management of patients with valvular heart disease: executive summary: a report of the American College of Cardiology/American Heart Association Task Force on Practice Guidelines. J Am Coll Cardiol. 2014; 63 (2014): 2438-88.

8. Nishimura RA, Otto CM, Bonow RO, Carabello BA, Erwin JP, Fleisher LA, et al. 2017 AHA/ACC focused update of the 2014 AHA/ACC Guideline for the Management of Patients With Valvular Heart Disease. J Am Coll Cardiol. 2017; 70: 252-89.

9. Kappetein AP, Head SJ, Généreux P, Piazza N, van Mieghem NM, Blackstone EH, et al. Updated standardized endpoint definitions for transcatheter aortic valve implantation: the Valve Academic Research Consortium-2 consensus document. Eur J Cardiothorac Surg. 2012; 42 (5): S45-60.

10. Capodanno D, Petronio AS, Prendergast B, Eltchaninoff $\mathrm{H}$, Vahanian A, Modine T, et al. Standardized definitions of structural deterioration and valve failure in assessing long-term durability of transcatheter and surgical aortic bioprosthetic valves: a consensus statement from the European Association of Percutaneous Cardiovascular Interventions (EAPCI) endorsed by the European Society of Cardiology (ESC) and the European Association for Cardio-Thoracic Surgery (EACTS). Eur Heart J. 2017; 38: 3382-90.

11. Thourani VH, Kodali S, Makkar RR, Herrmann HC, Williams M, Babaliaros V, et al. Transcatheter aortic valve replacement versus surgical valve replacement in intermediate-risk patients: a propensity score analysis. Lancet 2016; 387: 2218-25.

12. Latib A, Maisano F, Bertoldi L, Giacomini A, Shannon J, Cioni $\mathrm{M}$, et al. Transcatheter vs surgical aortic valve replacement in intermediate-surgical-risk patients with aortic stenosis: a propensity score-matched case-control study. Am Heart J. 2012; 164: 910-7.

13. Tamburino C, Barbanti M, D’Errigo P, Ranuci M, Onorati F, Covello RD, et al. 1-Year outcomes after transfemoral transcatheter or surgical aortic valve replacement: Results from the Italian OBSERVANT study. J Am Coll Cardiol. 2015; 66: 804-12.

14. Piazza N, Kalesan B, van Mieghem N, Head S, Wenaweser P, Carrel TP, et al. A 3-center comparison of 1-year mortality outcomes between transcatheter aortic valve implantation and surgical aortic valve replacement on the basis of propensity score matching among intermediate-risk surgical patients. JACC Cardiovasc Interv. 2013; 6: 443-51.

15. Sardar P, Kundu A, Chatterjee S, Feldman DN, Owan T, Kakouros N, et al. Transcatheter versus surgical aortic valve replacement in intermediate-risk patients: evidence from a meta-analysis. Catheter Cardiovasc Interv. 2017; 90: 504-15.

16. Gada H, Vora A, Millan-Iturbe O, Sztejfman M, Tumelero R, Gutiérrez L. Reproducibility of Cusp Overlap Technique to Reduce Permanent Pacemaker Implantation With Evolut: The Latin American Experience. J Am Coll Cardiol. 2020; 76 (17): Suppl B 202.

17. Gada H, Vora A, Siddique S, Wert Y, Michev I, Piazza $\mathrm{N}$, et al. Reduction of Rates of Permanent Pacemaker Implantation With 34-MM Evolut R Using Cusp Overlap Technique. J Am Coll Cardiol. 2020; 76 (17): Suppl B196.

18. Vemulapalli S, Holmes DR, Dai D, Matsouaka R, Mack MJ, Grover FL, et al. Valve hemodynamic deterioration and cardiovascular outcomes in TAVR: A report from the STS/ACC TVT Registry. Am Heart J. 2018; 195: 1-13.

19. Holy EW, Kebernik J, Abdelghani M, Stämpfli SF, Hellermann J, Allali A, et al. Long-term durability and hemodynamic performance of a self-expanding transcatheter heart valve beyond 5 years after implantation: A prospective observational study applying the standardized definitions of structural deterioration and valve failure. EuroIntervention 2018; 14 (4): e390-6. 